\title{
The Effect of the Radius of Covalent Bond on Melting Temperature of Organic Compounds
}

\author{
Ajeel F. A. Al-Zafeeri \\ The Public Authority For Applied Education And Training \\ The Higher Energy Institute -Chemical Processes Dept. Kuwait
}

\begin{abstract}
This paper presents a discussion of the effects of covalent radius value in producing new organic compounds, the value of covalent bond radius will be changed either by increasing or decreasing and hence a new organic compounds or new properties organic compounds will be created. It is found that the value of the covalent radius has really considerable effects on changing organic compounds properties such as melting point temperature.
\end{abstract}

Keywords: - Organic Compounds, Covalent Radius, Chemical Bonds, Organic Compounds Properties, Thermodynamics.

\section{INTRODUCTION}

The stability of a covalent bond results from a large quantity of electron density in the bonding region, the space between the two nuclei. In the bonding region, the electrons are nearly close to both nuclei, resulting in a lowering of the overall energy .The bonding electrons also mask the positive charges of the nuclei, so the nuclei do not make repulsion for each other as much as they would otherwise. There is always an optimum distance for the two bonded nuclei .If such nuclei are too far apart, their attraction for the bonding electrons is diminished. If such nuclei are too close together, their electrostatic repulsion pushes them apart .The internuclear distance where attraction and repulsion are balanced, which also gave the minimum energy (the strongest bond), is called the bond length. See fig.1, [9].

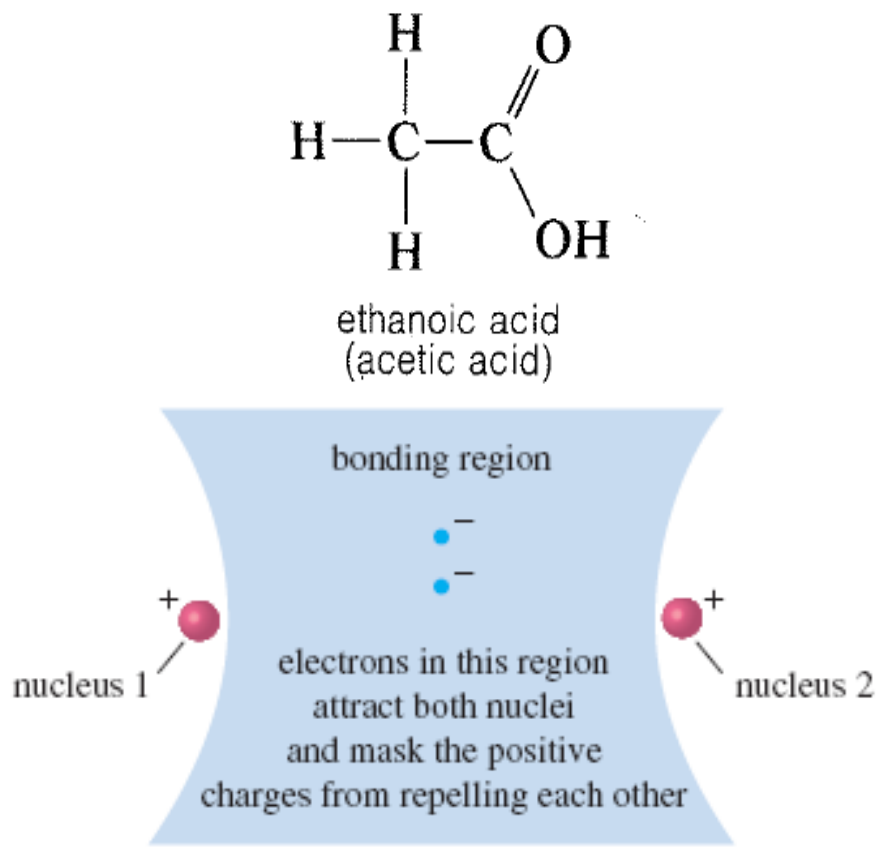

Fig. 1 bonding region between two atoms, [9].

Little of researches discussed the effects of covalent radius value in producing new organic compounds properties, Chung C. et. al., in this work a series of organic ammonium ions were exchanged onto clay minerals montmorillonite and hectorite. Thermal effects on these surface modified organic-inorganic complexes were investigated by using the differential thermal analysis and heating-oscillating X-ray diffraction methods. The researcher found that the organo-clay complexes were dehydrogenated at temperatures from 180 to $350{ }^{\circ} \mathrm{C} \sim$ 
depending on the organic cation used .Following the dehydrogenation, hydrolysis was found that it can take place leaving a layer of "carbon "on the clay surface. The "carbon "was then oxidized at a temperature of about $550^{\circ} \mathrm{C}$. Investigation of possible correlations between the boiling point, ionization potential, molecular structure activation energy, dehydrogenation and basicity, and carbon atoms bond distances of the organic cations showed that the geometric factors outweighed the acid, some chemical and electronic factors in determining the effect of montmorillonite and hectorite toward the dehydrogenation of organic ions were exchanged on the surfaces of these clay minerals, [2]. Nicholas J. Turro, exchanging the supramolecular organic photochemistry from softmatter hosts (micelles) and hard-matter hosts (porous solids) were discussed with an emphasis on how noncovalent interactions, which are at the heart of supramolecular chemistry, can be systematically exploited to control the catalytic and magnetic effects on the formation of covalent bonds from photochemically produced pairs of radical, [3].

Steven S., et al. Discussed the solid-phase measurements which are typically made using solvent extraction or thermal headspace analysis. The high temperatures and chemical solvents associated with such methods can modify the physical structure of polymeric materials and consequently affect mass transfer characteristics . To measure solid-phase concentrations under conditions resembling those in which the material would be installed in an indoor environment, a new technique for measuring volatile organic compounds concentrations in vinyl flooring (VF) and similar materials were developed. A $0.09 \mathrm{~m}^{2}$ section of new vinyl flooring was punched randomly to produce approximately $2000.78-\mathrm{cm}^{2}$ disks . The disks were milled to a powder at $-140^{\circ} \mathrm{C}$ to simultaneously homogenize the material and reduce the diffusion path length without loss of volatile organic compounds. volatile organic compounds were extracted from the vinyl flooring particles at room temperature by fluidized bed desorption and by direct thermal desorption at elevated temperatures. The volatile organic compounds in the extraction gas from fluidized bed desorption and direct thermal desorption were collected on sorbent tubes and analyzed by gas chromatography/mass spectrometry .Seven volatile organic compounds emitted by vinyl flooring were quantified .Concentration measurements by fluidized bed desorption ranged from $5.1 \mu \mathrm{g}-1 \mathrm{~g}$ vinyls flooring for $\mathrm{n}$-hexadecane to $130 \mu \mathrm{g}-1 \mathrm{~g}$ vinyls flooring for phenol . Concentrations measured by direct thermal desorption were higher than concentrations measured by fluidized bed desorption. Differences between fluidized beds desorption and direct thermal desorption results may be explained using free-volume/dual-mobility sorption theory, [5].

B. M. Mikhailov, discussed the covalent radii of carbon in the $s p^{2}$ and $s p$ and stated that their values are ranging from 0.73 and $0.69 \mathrm{~A}$, respectively, also the phenomenon of hyper conjugation of the first order $(\sigma, \pi$ conjugation) really does not exist. Also the researcher found that the shortening of such single carbon-carbon bond adjacent to a multiple bond is due not to the displacement of electrons by hyper conjugational (resonancemesomerism) mechanism, but it refers to an orbital effect: it is also found that a reduction in the covalent radii of carbon with increase in the contribution of bonding orbitals of this type. The author found that in molecules containing alternating multiple bonds the delocalization of $\pi$-electrons and leveling out of lengths of bonds do not occur. In such molecules the mesomeric (conjugation) effect (static effect of $\pi, \pi$ conjugation) postulated by resonance-mesomerism theory does not appear. The shortening of single bonds observed in molecules containing alternating multiple bonds is due to an orbital effect, [1]. Nicholas J. Turro, Exemplars of supramolecular organic photochemistry from soft-matter hosts (micelles) and hard-matter hosts (porous solids) were discussed with an emphasis on how non-covalent interactions, which are at the heart of supramolecular chemistry, can be systematically exploited to control the catalytic and magnetic effects on the formation of covalent bonds from photochemically produced pairs of radicals.[10].

Methods and materials

Covalent radius in organic compounds has an important role in producing new properties for such compounds; hence new compounds are resulted. Covalent radius variations in organic structures contributing in producing new compounds. In this work a virtual model is constructed to show the effects of covalent radius in properties of organic compounds.

Let us have the two different organic structures (figure 2).
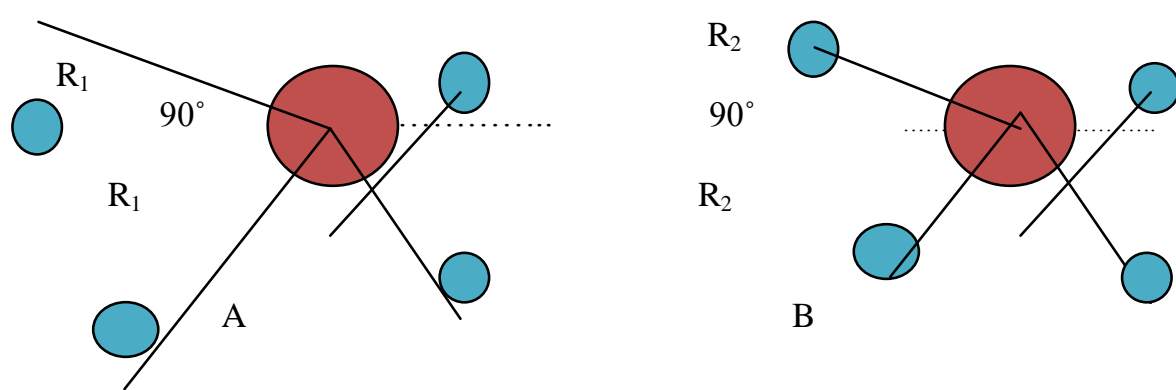
Fig. 2 Same organic compounds (A, and B) with same structure different covalent radii From the two organic configurations it is clear that distance between the two sub atoms (colored in blue) is differ from the first construction (A) and (B). In A the distance is equal to

$D_{1}=\sqrt{2 R_{1}^{2}}$

While in the second configuration the distance can be given as

$D_{2}=\sqrt{2 R_{2}^{2}}$

\section{RESULTS AND DISCUSSION}

Since $R_{1}$ is greater than $R_{2}$ then $D_{2}$ is less than $D_{1}$. This difference in distance makes the properties of same organic compounds different either in boiling point, melting point, tensile strength and other mechanical properties, since the total length and width of the organic compounds will be differed. It is found that the distances between the sub-atoms in the organic compounds affected by the covalent radius i.e as the covalent radius increases the distance and the total length of the compound increase, and hence the properties of the organic compound change. Melting points, boiling point, tensile strength of such organic compounds are decrease as the length of the compound increases i.e. as D increases such that

$[\mathrm{M}, \mathrm{B}, \mathrm{T}]=[\mathrm{M} 0, \mathrm{~B} 0, \mathrm{~T} 0]-\mathrm{C}(\mathrm{D})$

where

$\mathrm{M}$ : is the melting point temperature, $\mathrm{B}$ : is the boiling temperature, $\mathrm{T}$ : is the tensile strength of the material. M0, B0, and T0: are the base values of the last properties respectively. C: is a constant, and D is the distance between atoms in the organic compounds. Such relations can be represented as in figure (3).

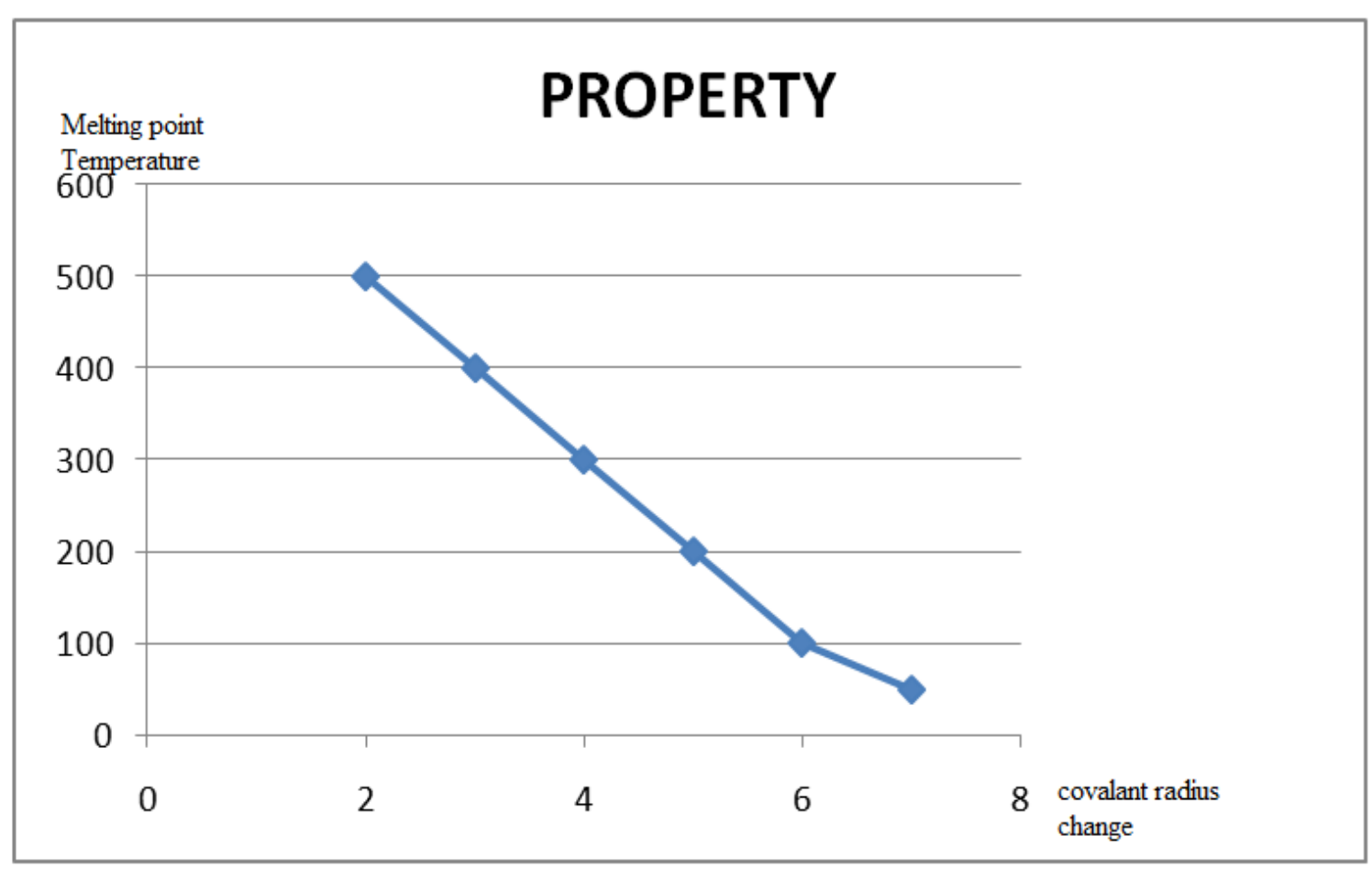

Fig. (3) Melting point variation with the covalent radius change.

Fig. 3 represents a demonstration for the properties of organic compounds as a relation with the atoms distance (D), it is clear that as the distance (D) increases the value of melting point, and also by the same behavior boiling point, and tensile strength of such compounds decrease.

\section{CONCLUSION}

The main conclusion can be drawn up from this discussion that to produce a new properties for organic compounds having the same monomers and same structure is by changing up the covalent bond radius, this can be made by changing up pressure, temperature, or any other parameter. This will produce some new properties for organic compounds. 


\section{REFERENCES}

[1]. B. M. Mikhailov, 2004, Covalent radii of carbon and the conjugation problem, Russian chemical bulletin, springer, vol. (8), no. (8), pp: 1284-1290.

[2]. CHUNG CHI CHOU and JAMES L .McATEE, Jr.1969, Thermal Decomposition Of Organo-ammonium Compounds Exchanged Onto Montmorillonite And Hectorit, Clays and Clay Minerals, 1969, Vol .17, pp . 339-346 .Pergamon Press .

[3]. Nicholas J .Turro, 2002, supramolecular organic photochemistry :Control of covalent bond formation through noncovalent supramolecular interactions and magnetic effects, PNAS _ April 16, 2002 _ vol .99 - no. 8 _ 4805-4809.

[4]. S.A.Abd El-Maksoud, 2008, The Effect of Organic Compounds on the Electrochemical Behavior of Steel in Acidic Media.A review, Int .J .Electrochem .Sci., 3: (528 -555).

[5]. Steven S .Cox, Alfred T .Hodgson, and John C .Little, 2002, Measuring Concentrations of Volatile Organic Compounds in Vinyl Flooring, LBNL-47086, Accepted by Journal of the Air \& Waste Management Association, 04/01.

[6]. Koros, W., Chan, A., and Paul, D .Sorption and transport of various gases in polycarbonate, Journal of Membrane Science, 1977, 2; 165-190.

[7]. Schwarzenbach, R.; Gschwend, P.M.; Imboden, D.M .Environmental Organic Chemistry; John Wiley \& Sons :New York, 1993; p.200.

[8]. Cox, S.S.; Little, J.C.; Hodgson, A.T .A New Method to Predict Emission Rates of Volatile Compounds from Vinyl Flooring, In Proceedings of Healthy Buildings 2000, Helsinki, Finland, 2000, 4; 169-174.

[9]. Graham S., and Craig F., 2000, Organic Chemistry, $7^{\text {th }}$ edition, John Wiley, pp: 110-150, 1-50.

[10]. Nicholas J. Turro, 2002, Supramolecular organic photochemistry: Control of covalent bond formation through non-covalent supramolecular interactions and magnetic effects, PNAS _ April 16, 2002 _ vol. 99 - no. 8 _ 4805-4809. 